\title{
Gu Hongming - vom Kulturvermittler zum Kulturheros?
}

\author{
Isabell Oberle
}

\section{Mittler im interkulturellen Transfer}

Interkulturelle Transferprozesse sind notwendigerweise auf Vermittlerfiguren oder -instanzen angewiesen. Nicht Nationalkulturen stehen als selbstständige Entitäten in wechselseitigem Austausch, sondern Einzelpersonen oder Institutionen. Will man interkulturellen Transfers in ihrer Dynamik gerecht werden, reicht es mithin nicht aus, Ausgangs- und Zielkontext komparatistisch zu untersuchen. Vielmehr müssen Akteur/innen und Medien beleuchtet werden, die dem Transfer eines Kulturgutes den Weg bereiten. Diese Erkenntnis liegt zwar schon der frühen Kulturtransferforschung der 1980er und 1990er Jahre zugrunde, ${ }^{1}$ jedoch wird die gezielte Beschäftigung mit Mittlerfiguren von Thomas Keller 2011 noch als Desiderat formuliert: „So wie der reale Rezipient in den Blick kommen muss, muss auch der reale Mittler wahrgenommen werden".2

Der vorliegende Beitrag greift die Forderung auf, indem er einen Mittler des deutsch-chinesischen Kulturfelds ins Zentrum rückt, nämlich den chinesischen Gelehrten und Kulturphilosophen Gu Hongming (" 1857, Penang; †1928, Shanghai). ${ }^{3}$ Heute nahezu vergessen, zählte er im Westen zu Beginn des 20. Jahrhunderts

1 Vgl. bspw. Michel Espagne: Die Rolle der Mittler im Kulturtransfer, in: Hans-Jürgen Lüsebrink / Rolf Reichardt (Hg.): Kulturtransfer im Epochenumbruch Frankreich - Deutschland 1770 bis 1815 (Deutsch-Französische Kulturbibliothek 9.1), Leipzig 1997, S. 309-329, hier S. 310-311, oder Bernd Kortländer: Begrenzung - Entgrenzung. Kultur- und Wissenschaftstransfer in Europa, in: Lothar Jordan / Bernd Kortländer (Hg.): Nationale Grenzen und internationaler Austausch. Studien zum Kulturtransfer in Europa (Communicatio. Studien zur europäischen Literatur- und Kulturgeschichte 10), Tübingen 1995, S. 1-19, hier S. 5-6. Zur selben Zeit entstanden auch erste Arbeiten zu Mittlerfiguren, insbesondere im deutsch-französischen Kulturfeld, wie Hans-Jürgen Lüsebrink / János Riesz (Hg.): Feindbild und Faszination. Vermittlerfiguren und Wahrnehmungsprozesse in den deutschfranzösischen Kulturbeziehungen (Schule und Forschung), Frankfurt am Main 1984, und Michel Espagne / Werner Greiling (Hg.): Frankreichfreunde. Mittler des französischdeutschen Kulturtransfers (1750-1850) (Deutsch-Französische Kulturbibliothek 7), Leipzig 1996.

2 Thomas Keller: Kulturtransferforschung: Grenzgänge zwischen den Kulturen, in: Stephan Moebius / Dirk Quadflieg (Hg.): Kultur. Theorien der Gegenwart, Wiesbaden 22011, S. 106-119, hier S. 117.

3 Gu Hongming (heutige Schreibweise) bzw. Ku Hung-Ming (damals gängige Schreibweise) ist der Volljährigkeitsname (,zi'), unter dem er seine Schriften in englischer Sprache publizierte. Sein Familienname (,míng') lautet T’ang-shêng (vgl. Uwe Riediger: Ku Hung-ming Umrisse eines Lebens (-1928), in: Oriens Extremus. Zeitschrift für Sprache, Kunst und Kultur der Länder des Fernen Ostens 31, 1987/88, S. 197-242, hier S. 206. Zur Biographie Gus, leider häufig mit widersprüchlichen Angaben, vgl. ebd., S. 205-226; Gotelind Müller: 
zu den wirkungsmächtigsten Denkern des Orients. ${ }^{4}$ Seine kulturkonservativen Schriften über die chinesische Geistes- und Lebenswelt wurden im Europa der 1910er und frühen 1920er Jahre rezipiert und zustimmend aufgenommen, am intensivsten wohl in den krisengeschüttelten deutschsprachigen Ländern seit dem Ersten Weltkrieg. Bisweilen mehr als nur verehrt, wurde Gu der Status eines Helden zuerkannt. Seine erstaunliche Karriere im Westen lässt sich nur erklären als ein Zusammenspiel aus den diskursiven Bedürfnissen der Aufnahmekultur, die über Auswahl und Adaption eines Imports maßgeblich mitbestimmten und dem Transfer Vorschub leisteten, ${ }^{5}$ einerseits und der Selbstinszenierung Gus andererseits, die sich an den Erwartungen der Zielkultur orientierte. Dies soll im Folgenden dargelegt werden. In einem ersten Schritt werden die Rezeption Gus in Deutschland und Österreich nachgezeichnet und die hinter dem Transfer wirksamen Beweggründe sowie die Funktionen der Heroisierung analysiert. Dabei geht es nicht so sehr darum, ob die Rezipient/innen Gus Werke so verstanden, wie er sie konzipiert hatte, als vielmehr um ihre Haltung ihm gegenüber. Im Anschluss werden Gus Strategien, sich im Westen Gehör zu verschaffen, anschlussfähig zu sein und $\mathrm{Zu}$ stimmung zu finden, betrachtet.

\section{Rezeption: Skepsis, Einverständnis, Glorifikation}

Im Westen ist Gu Hongming in erster Linie als ein Vertreter alter chinesischer Kultur, insbesondere des Konfuzianismus wahrgenommen worden. Seine Beschäftigung mit der chinesischen Geisteswelt setzte allerdings erst ein, als $\mathrm{Gu}$, der sich lange in Europa aufgehalten hatte - bis 1877 studierte er unter anderem Englische Literatur in Schottland und reiste in den beiden folgenden Jahren nach Deutsch-

Gu Hongming (1857-1928) und Chinas Verteidigung gegen das Abendland, in: Orientierungen. Zeitschrift zur Kultur Asiens 18.1, 2006, S. 1-23, hier S. 4-8; Chunmei Du: Gu Hongming as a Cultural Amphibian: A Confucian Universalist Critique of Modern Civilization, in: Journal of World History 22.4, 2011, S. 715-746, hier S. 716-722; ders.: Gu Hongming's Eccentric Chinese Odyssey, Philadelphia 2019, S. 2-9; Xiaogiao Wu: Ku HungMing und der Kulturdialog zwischen China und Europa im 20. Jahrhundert, in: Internationale Kulturwissenschaften, 1999, www.inst.at/studies/s_0712_d.htm, 23. Mai 2019.

4 Gus Prominenz nicht nur unter deutschen bzw. deutschsprachigen Autoren bezeugt schon eine Recherche in der Datenbank China and the West (1245-2000) der Universität Zürich: www.aoi.uzh.ch/de/sinologie/forschung/chinaundderwesten.html, 30. August 2019. Die Datenbank listet Rezeptionsbelege u. a. bei Hermann Hesse, Walter Benjamin, Rudolf Pannwitz, William Somerset Maugham und Leo Tolstoi. Gus Rezeption wurde bisher nie umfänglich untersucht. Kursorische Überblicke oder punktuelle Ausschnitte bieten: Riediger: Ku Hung-Ming (Anm. 3), S. 198-202; Müller: Gu Hongming (Anm. 3), S. 9-16; Du: Gu Hongming's Eccentric Chinese Odyssey (Anm. 3), S. 46-67.

5 Vgl. Espagne: Die Rolle der Mittler im Kulturtransfer (Anm. 1), S. 310. Zu den Interessen der Aufnahmekultur, von denen Transferprozesse wesentlich getragen werden, vgl. Kortländer: Begrenzung - Entgrenzung (Anm. 1), S. 7. 
land und Frankreich ${ }^{6}$-, sich Mitte der 1880er Jahre im chinesischen Wuhan, später auch in Peking und Shanghai, ansiedelte. ${ }^{7}$ Seitdem arbeitete er eifrig an der Verbreitung von Wissen über die chinesische Philosophie im Westen. Er übertrug konfuzianische Standardwerke ins Englische, etwa The Discourses and Sayings of Confucius (1989) und The Universal Order, or Conduct of Life (1906). ${ }^{8}$ Seine theoretischen Schriften, die er in englischer Sprache veröffentlichte, sodass sie einem westlichen Publikum zugänglich waren, sollten diesem Einblicke in die traditionelle chinesische Kultur wie auch in moderne Entwicklungen des Landes insbesondere in der Auseinandersetzung mit westlichen Einflüssen geben. ${ }^{9}$ In Europa machten $\mathrm{Gu}$ zwei Aufsatzsammlungen, The Story of a Chinese Oxford Movement (1910) und The Spirit of Chinese People (1915), bekannt. In beiden Veröffentlichungen kritisierte Gu den westlichen Imperialismus und Kolonialismus sowie die an westlichen Ideen geschulten, modernen Entwicklungen in China, da sie die wahre chinesische Kultur zerstören würden. Erstens wollte er die eigene chinesische Identität und Kultur vor dem Einfluss des militaristisch-materialistischen Westens bewahren, was ihm zufolge nur durch die Besinnung auf moralisch-konfuzianische Werte gelingen könne. Zweitens strebte er an, die westliche Welt in traditioneller chinesischer Kultur zu unterweisen. Er präsentierte ihr den Konfuzianismus als Mittel, um dem von ihm monierten Sittenverfall durch Imperialismus, Militarismus und Materialismus entgegenzuwirken. ${ }^{10}$

In Deutschland wurde die Rezeption Gus eingeleitet mit der von Alfons Paquet angeregten ${ }^{11}$ und 1911 von Richard Wilhelm besorgten Übersetzung von The Story

6 Aus den Bemerkungen Richard Wilhelms über Gu geht hervor, dass dieser Englisch, Deutsch und Französisch sprach und schrieb (vgl. Richard Wilhelm: Die Seele Chinas, Berlin 1926, S. 174-176).

7 Die Auseinandersetzung mit und Hinwendung zur chinesischen Kultur wurde maßgeblich angeregt durch ein Treffen mit dem chinesischen Gelehrten und Beamten Ma Jianzhong, der sein Studium ebenfalls im europäischen Ausland verbracht hatte (vgl. Du: Gu Hongming's Eccentric Chinese Odyssey [Anm. 3], S. 89-90; Müller: Gu Hongming [Anm. 3], S. 5-6; Riediger: Ku Hung-ming [Anm. 3], S. 208-209).

8 Vgl. Mingguo Zhong: Self-Orientalization and its Counteraction against the Cultural Purpose of Gu Hongming in His Discourses and Sayings of Confucius, in: Theory and Practice in Language Studies 2.11, 2012, S. 2417-2421, hier S. 2417. Die von Europäern angefertigten Übersetzungen hielt er für unzulänglich (vgl. Wu: Ku Hung-Ming und der Kulturdialog [Anm. 3], o. A.).

9 „Der Gegenstand dieses Buches ist, den Geist der chinesischen Zivilisation zu erklären und ihren Wert zu zeigen", heißt es explizit im Vorwort von The Spirit of Chinese People (Ku Hung-Ming: Der Geist des chinesischen Volkes \& Der Ausweg aus dem Krieg, übers. von Oscar Adolf Hermann Schmitz, Jena 1916, S. 9).

$10 \mathrm{Zu}$ den beiden Schriften vgl. Du: Gu Hongming as a Cultural Amphibian (Anm.3), S. 723; ders.: Gu Hongming's Eccentric Chinese Odyssey (Anm. 3), S. 25-26; Müller: Gu Hongming (Anm.3), S. 13; Riediger: Ku Hung-ming (Anm.3), S. 223; Wu: Ku HungMing und der Kulturdialog (Anm. 3), o. A. Eine ausführliche Analyse von Gus ideologischem Standpunkt sowie seiner Heranbildung legte jüngst Du: Gu Hongming’s Eccentric Chinese Odyssey (Anm. 3) vor.

11 Vgl. Alfons Paquet: Vorwort, in: Ku Hung-Ming: Chinas Verteidigung gegen europäische Ideen. Kritische Aufsätze, übers. von Richard Wilhelm, Jena 1911, S. i-xiv, hier S. xiv. 
of a Chinese Oxford Movement, der den Titel in Chinas Verteidigung gegen europäische Ideen umwidmete. The Spirit of Chinese People wurde 1916 als Der Geist des chinesischen Volkes von Oscar Adolf Hermann Schmitz, damals ein populärer Gesellschaftsschriftsteller und Mitglied der Münchner Bohème, ins Deutsche übersetzt. 1920 übertrug Heinrich Nelson, Jurist und Vater des Moralphilosophen Leonhard Nelson, die weniger bekannte Aufsatzsammlung über den Ersten Weltkrieg Vox clamantis: Essays on the War and Other Subjects (1918), unter dem Titel Vox clamantis. Betrachtungen über den Krieg und anderes. In den Vorworten loben Paquet (in der Ausgabe Wilhelms), Schmitz und Nelson allesamt Gus Einblicke in chinesische Kultur und Gesellschaft, seine Kenntnisse von und Sicht auf Europa sowie seinen Einsatz für eine konfuzianische Werteordnung in China. ${ }^{12}$ Mit der Würdigung Gus behaupten sie dessen bedeutsamen Stellenwert in zeitgenössischen Diskursen und rechtfertigen die Verbreitung seiner Werke im deutschen Sprachraum und damit ihre eigene übersetzerische Tätigkeit.

Wie die Publikationsdaten der Übersetzungen anzeigen, fiel die Rezeption Gus in die 1910er und frühen 1920er Jahre. Sie schlägt sich indes nicht nur in Übersetzungen und überwiegend positiven Rezensionen, auch von bedeutenden Schriftstellern wie Hermann Hesse, ${ }^{13}$ nieder. Gu findet darüber hinaus Erwähnung in Egodokumenten wie Briefen und Reiseerinnerungen. Richard Wilhelm berichtet von ihm in Die Seele Chinas (1926), Alfons Paquet in Li oder Im neuen Osten (1912) und Hermann Graf Keyserling im Reisetagebuch eines Philosophen (1919). ${ }^{14}$ Hugo von Hofmannsthal und Rudolf Pannwitz tauschten sich brieflich über ihn und seine Werke aus, ebenso Walter Benjamin. ${ }^{15}$ Nicht zuletzt bemühten deutsche und österreichische Intellektuelle Gus Studien in eigenen theoretischen Reflexionen und es-

12 Vgl. ebd., S. i-ii und xi; Oscar Adolf Hermann Schmitz: Zur Einführung, in: Ku HungMing: Der Geist des chinesischen Volkes \& Der Ausweg aus dem Krieg, übers. von Oscar Adolf Hermann Schmitz, Jena 1916, S. 1-5, hier S. 2; Heinrich Nelson: Geleitwort des Übersetzers, in: Ku Hung-Ming: Vox Clamantis. Betrachtungen über den Krieg und anderes, übers. von Heinrich Nelson, Leipzig 1920, S. 3-6, hier S. 3-5.

13 Hermann Hesse: Chinas Verteidigung gegen europäische Ideen, in: Volker Michels (Hg.): Hermann Hesse. Die Welt im Buch. Leseerfahrungen II: Rezensionen und Aufsätze aus den Jahren 1911-1916, Frankfurt am Main 1998, S. 78-79; außerdem Christoph Behm: Ein chinesischer Spiegel, in: Die neue Rundschau 23.1, 1912, S. 863-868, oder Wolf von Dewall: Ku Hung-Ming: Der Geist des chinesischen Volkes und der Ausweg aus dem Krieg [Rez.], in: Weltwirtschaftliches Archiv 11, 1917, S. 370-378.

14 Wilhelm: Die Seele Chinas (Anm. 6), S. 159-178, bes. S. 174; Alfons Paquet: Li oder Im Neuen Osten, Frankfurt am Main 1912, S. 289-292 (nahezu wörtliche Beschreibung in: ders.: Vorwort [Anm.11], S. xi-xiv, sowie ders.: Chinesische Schriftsteller, in: März. Eine Wochenschrift 5.3, 1911, S. 464-472 und S. 508-513, hier S. 467-472); Hermann Graf Keyserling: Die gesammelten Werke, Bd. 1: Das Reisetagebuch eines Philosophen, Darmstadt/Baden-Baden 1956, S. 391-394.

15 Gerhard Schuster (Hg.): Hugo von Hofmannsthal. Rudolf Pannwitz. Briefwechsel 19071926, Frankfurt am Main 1996, S. 12-13 und S. 449-456; Christoph Gödde / Henri Lonitz (Hg.): Walter Benjamin. Gesammelte Briefe, Bd. 1: 1910-1918, Frankfurt am Main 1995, S. 77-78. 
sayistischen Arbeiten, wie Pannwitz in Die Krisis der europäischen Kultur (1917), ${ }^{16}$ Hofmannsthal in seinen Reden in Skandinavien (1916) und seiner Berner Rede Über die europäische Idee (1917), ${ }^{17}$ ferner der Moralphilosoph Leonhard Nelson und sein Anhänger Hans Mühlestein. ${ }^{18}$ Ihnen allen war indes weniger an einer Unterweisung in konfuzianischer Philosophie um der chinesischen Kultur willen gelegen. Vielmehr sahen sie in Gus Schriften eine Diagnose der eigenen, krankenden Kultur sowie ein mögliches Gegenmittel zur Kurierung. ${ }^{19}$ Damit decken sich ihre Einschätzungen mit dem vorherrschenden Chinabild der Zeit. In Europa hatte sich um 1900 ein ausgesprochenes Krisenbewusstsein entwickelt, das mit einem neu erwachten Interesse für andere Kulturräume und fremde Gesellschaftsentwürfe einherging. Der radikale Fortschritt von Technik und Industrie, der im ausgehenden 19. Jahrhundert noch begrüßt und als Ursache der Verbesserung machtpolitischer wie sozioökonomischer Verhältnisse wahrgenommen worden war, verlor mit der Jahrhundertwende seinen Nimbus: Technisierung, Rationalisierung und Urbanisierung sowie die Übermacht des Materialismus und der im Zuge des Krieges entfesselte Militarismus lösten ein Gefühl der Besorgnis aus. Insbesondere Vertreter der konservativen und gebildeten Schichten fürchteten einen Verfall moralischsittlicher wie kultureller Werte und eine Veräußerlichung des menschlichen Lebens. Sie wandten sich dem Fernen Osten zu, der die Bewahrung altwürdiger Traditionen sowie die Alleinheit mit der Natur versprach. ${ }^{20}$ China wurde im Zuge dessen nicht mehr wie noch im 19. Jahrhundert als die ,Gelbe Gefahr' verunglimpft, sondern avancierte zum nachahmenswerten Vorbild. Im Zentrum stand gleichwohl

16 Rudolf Pannwitz: Werke, Bd. 2: Die Krisis der europäischen Kultur, München-Feldafing 1921, S. 226-261.

17 Klaus E. Bohnenkamp u. a. (Hg.): Hugo von Hofmannsthal. Sämtliche Werke, Bd. 34: Reden und Aufsätze 3, Frankfurt am Main 2011, S. 290-324 (Aufzeichnungen zu Reden in Skandinavien) und S. 324-335 (Über die europäische Idee).

18 Leonard Nelson: Gesammelte Schriften in neun Bänden, Bd. 8: Sittlichkeit und Bildung, Hamburg 1971, S. 277-302 (Ethischer Realismus) und S. 497-522 (Erziehung zum Führer); Hans Mühlestein: Europäische Reformation. Philosophische Betrachtungen über den moralischen Ursprung der politischen Krisis Europas, Leipzig 1918.

19 Vgl. dazu auch die Einleitung in Adolf Reichweins zeitgenössischer Dissertationsarbeit (erschienen 1923): China and Europe. Intellectual and Artistic Contacts in the XVIII ${ }^{\text {th }}$ Century, London/New York 1996, S. 3-11.

20 Vgl. Ki-Chung Bae: Chinaromane in der deutschen Literatur der Weimarer Republik (Wissenschaftliche Beiträge aus dem Tectum Verlag, Reihe Germanistik 5), Marburg 1999, S. 11-13; Ruixin Han: Die China-Rezeption bei expressionistischen Autoren (Europäische Hochschulschriften 1421), Frankfurt am Main 1993, S. 30-45; Helmut Fries: Die große Katharsis. Der Erste Weltkrieg in der Sicht deutscher Dichter und Gelehrter, Bd. 1: Die Kriegsbegeisterung von 1914: Ursprünge - Denkweisen - Auflösung, Konstanz 1994, S. 11-138; Volker Drehsen / Walter Sparn: Die Moderne: Kulturkrise und Konstruktionsgeist, in: Volker Drehsen / Walter Sparn (Hg.): Vom Weltbildwandel zur Weltanschauungsanalyse. Krisenwahrnehmung und Krisenbewältigung um 1900, Berlin 1996, S. 11-29, hier S. 11-16. 
nicht das zeitgenössische China - auch die Xinhai-Revolution von 1911 interessierte nur bedingt -, sondern das Stereotyp des klassischen, geistigen China. ${ }^{21}$

Stellvertretend für die zahlreichen Rezeptionszeugnisse, die dieselbe Tendenz aufweisen, sei Rudolf Pannwitz' Essay Die Krisis der europäischen Kultur, erschienen während des Ersten Weltkriegs 1917, angeführt, anhand dessen sich symptomatisch die Haltung gegenüber China und Gu Hongming aufzeigen lässt. Der deutsche Schriftsteller und Philosoph konstatiert eine durch Nationalismus und Materialismus hervorgerufene Krise der Moderne, die ganz Europa erfasst habe und nur durch eine Hinwendung zur traditionellen ostasiatischen Kultur überwunden werden könne:22

Einen ausweg aus der krisis der europäischen kultur auf irgend einem begangnen wege gibt es nicht mehr [...] für alle und keinen für einen und alle ist der weg eine verschmelzung unserer unter sich verschmolzenen europäischen halbkulturen mit den groszen orientalischen klassischen kulturen. geschehe wieder was immer geschehn dass die jüngeren völker scheinbar die älteren kolonisieren wahrhaft von ihnen sich kolonisieren lassen! kultur ist schlechthin etwas orientalisches etwas aussereuropäisches[.] $]^{23}$

Es seien die ethisch-moralischen Werte, so Pannwitz weiter, die Europa verloren gegangen seien, und als ausgereifte, Sittenlehren' müssten Buddhismus, Daoismus und insbesondere Konfuzianismus zu neuen Modellen für die Europäer werden. ${ }^{24}$ In Pannwitz' Augen verbürgte Gu ebendieses Weltbild. Seine Ausführungen zu Europa konnten für ihn treffender nicht sein. Er galt ihm als „wahrscheinlich der einzige der über den europäischen krieg und europa wertvolles sagt“. ${ }^{25}$ Auch Hofmannsthal nutzte Gu gelegentlich als Gewährsmann seiner Kulturkritik, wie er Pannwitz in einem Brief berichtet. ${ }^{26}$ Als er während seiner Reisen in Skandinavien 1916 „von den Studenten aufgefordert, etwas über diese gemeinsame Not zu sagen“, wusste er nichts anderes, „als von dem Buch des Ku-hung-ling auszuge-

21 Vgl. Wolfgang Bauer: Die Rezeption der chinesischen Literatur in Deutschland und Europa, in: Günther Debon (Hg.): Neues Handbuch der Literaturwissenschaft, Bd. 23: Ostasiatische Literaturen, Wiesbaden 1984, S. 159-192, hier S. 175 und S. 184, sowie Han: Die China-Rezeption bei expressionistischen Autoren (Anm. 20), S. 30.

22 Vgl. dazu Paul M. Lützeler: Publizistische Germanistik: Essays und Kritiken, Berlin/ Boston 2015, S. 317-318.

23 Pannwitz: Die Krisis der europäischen Kultur (Anm. 16), S. 226.

24 Vgl. ebd., S. 227-228. Pannwitz' Vision der Gegenwart ist maßgeblich von der Erfahrung des Ersten Weltkriegs und damit dem Verlust eines friedlichen Europas geprägt. Die Hinwendung zu Ostasien reicht gleichwohl bis in den Beginn des 20. Jahrhunderts zurück. Verwiesen sei auf Die Briefe des Zurückgekehrten (1907) von Hugo von Hofmannsthal, mit dem Pannwitz in engem brieflichem Austausch stand; vgl. Thomas Pekar: Hofmannsthals ,Umweg über Asien‘. Zur Konstellation von Europa und Asien im europäischen ,KrisenDiskurs' am Anfang des 20. Jahrhunderts, in: Deutsche Vierteljahrsschrift für Literaturwissenschaft und Geistesgeschichte 83, 2009, S. 246-261, hier S. 259. Pannwitz: Die Krisis der europäischen Kultur (Anm. 16), S. 245.

26 Vgl. Pekar: Hofmannsthals ,Umweg über Asien` (Anm. 24), S. 250-251; Hartmut Zelinsky: Hugo von Hofmannsthal und Asien, in: Roger Bauer u. a. (Hg.): Fin de siècle. Zu Literatur und Kunst der Jahrhundertwende (Studien zur Philosophie und Literatur des 19. Jahrhunderts 35), Frankfurt am Main 1977, S. 508-566, hier S. 511. 
hen“ ${ }^{27}$ In seinen Notizen zu seiner ein Jahr später in Bern gehaltenen, modernekritischen Rede Über die europäische Idee beruft sich Hofmannsthal auf Gus „Verurteilung des europäischen Wesens, umso zermalmender als sie würdevoll und ohne Polemik ist“. ${ }^{28}$

Pannwitz geht über Hofmannsthal hinaus, indem er nicht nur Gus Europakritik teilt, sondern auch dessen Lösungsvorschlag, der in einer Orientierung an den moralischen Maximen des Konfuzianismus bestehe:

trotzdem sieht er das ganz entscheidende die krisis der europäischen kultur als tatsache und ihre letzten ursachen richtiger als die europäer heute und gibt weite strecken hin endgültige lösungen [...] damit dass wir keinen anderen weg haben als die reine sittlichkeit-religion des kongfutse um aus unserer sogenannten sozialen frage herauszukommen wird er einfach recht haben[. $]^{29}$

Gus kontrastiver Gegenüberstellung Europas und des chinesischen Konfuzianismus liegt eine kulturbewahrende Stoßrichtung zugrunde, die in den Rezeptionszeugnissen stets hervorgehoben und gelobt wird: Walter Benjamin bemerkt - positiv überrascht - einen nur selten anzutreffenden „radikalen Kulturwillen“, 30 Rudolf Pannwitz bewundert Gus „lautere absichten mit der kultur“, ${ }^{31}$ und Heinrich Nelson würdigt Gus Erkenntnis, „daß es für die Völker des Ostens zu ihrer Selbsterhaltung notwendig ist, fest auf dem Boden der eigenen uralten bewährten Kultur stehen zu bleiben und sich nicht die auf ganz andere Verhältnisse zugeschnittene westliche Kultur aufdrängen zu lassen“. 32

Das Motiv für die Rezeption Gus bei deutschen und österreichischen Intellektuellen, die kulturkonservativen Kreisen zuzurechnen sind, lässt sich mit Bert Kortländer als ideologisches Interesse beschreiben. Dieses drückt sich aus in „Normen und Werten, die durch die importierten Kulturgüter in ihrer Ursprungskultur tatsächlich vertreten werden oder durch solche Normen und Werte, die die importierten Güter nach der Absicht der Importeure repräsentieren sollen“. ${ }^{33}$ Pannwitz und andere berufen sich deshalb auf $\mathrm{Gu}$, weil er sich für eine Überzeugung einsetzt, die auch die ihre ist, und weil er als Vertreter der fremden Kultur leicht mit ihr in eins gesetzt werden kann. Schon 1911 wird Gu von Paquet als „chinesischer Lite-

27 Hugo von Hofmannsthal an Rudolf Pannwitz (29.7.1917), in: Schuster: Briefwechsel (Anm. 15), S. 12-13, hier S. 12.

28 Hugo von Hofmannsthal: Über die europäische Idee, in: Bohnenkamp u. a. (Hg.): Reden und Aufsätze 3 (Anm. 17), S. 324-335, hier S. 327.

29 Pannwitz: Die Krisis der europäischen Kultur (Anm. 16), S. 247-248; zu Pannwitz' ungetrübter Sicht auf Gu vgl. auch Ingrid Schuster: China und Japan in der deutschen Literatur 1890-1925, Bern 1977, S. 164-165.

30 Walter Benjamin: An Ludwig Strauss, in: Gesammelte Briefe, Bd. 1 (Anm. 15), S. 77-78, hier S. 77.

31 Pannwitz: Die Krisis der europäischen Kultur (Anm. 16), S. 157.

32 Nelson: Geleitwort des Übersetzers (Anm. 12), S. 3-4.

33 Kortländer: Begrenzung - Entgrenzung (Anm. 1), S. 7. Davon zu unterscheiden sind das technische sowie das praktische Interesse. Ähnlich auch Espagne: Die Rolle der Mittler im Kulturtransfer (Anm. 1), S. 313. 
rat von selbstständigem und starkem Denken voll innerer Beziehung zu dem klassischen Lehrer seines Volkes“34 klassifiziert - eine Einschätzung, die immer wieder aufgegriffen wurde: Hesse bezeichnet ihn als „ein[en] feine[n], gescheite[n] Vertreter der alten chinesischen Kultur und Moral“, 35 für Pannwitz ist er ein „reiner vertreter der klassischen chinesischen kultur" und ein „ganz edle[r] und lautere[r] nachfolger des kongfutse“, 36 und Mühlestein sieht in ihm einen „moderne[n] Apostel des Konfuzius “ ${ }^{37}$ Durch seine Stilisierung zum einlinigen Apologeten des Konfuzianismus - auch mithilfe christlichen Vokabulars - erhält Gu den Status eines Eingeweihten in göttliches Wissen, das ihn von der Masse abhebt und heroisiert. Die Heroisierung dient in diesem Fall dazu, gegen Kritik zu immunisieren, Gus Weltanschauung zu legitimieren und die Verbindlichkeit seiner Überlegungen zu begründen. Ihren stärksten Ausdruck findet die Verehrung Gus in den Arbeiten der Moralphilosophen, die mit $\mathrm{Gu}$ die eigenen neokantianischen, universalistischen Thesen untermauern, etwa Leonhard Nelson in Erziehung zum Führer (1920) und Ethischer Realismus (1921). ${ }^{38}$ Seinem Buch Reformation der Gesinnung von 1917 stellt er sogar ein Zitat aus Gus Chinas Verteidigung gegen europäische Ideen voran. ${ }^{39}$ Der Schweizer Hans Mühlestein zitiert Gu weitaus umfassender, verweist in Fragen moralischen Handelns auf dessen Erläuterungen zu Konfuzius und macht sich dessen Standpunkt über die Krise der europäischen Kultur zu eigen. ${ }^{40}$ Sein 1918 erschienenes Buch Europäische Reformation, das viele der Zitate enthält, widmet er Gu „[z]um Zeichen der Verehrung und des Einverständnisses“. ${ }^{41}$

Die Heroisierung Gus scheint noch einem anderen Zweck zu dienen, da Kritiker dem reflexiven Niveau seiner Arbeiten durchaus auch missbilligend gegenüberstanden. So misst der insgesamt positiv gestimmte Hermann Hesse den theoretischen Erwägungen doch kaum Bedeutung bei, er hält sie für einseitig und wenig originell. ${ }^{42}$ Der kritisch eingestellte Historiker Ludwig Rieß bemängelt in der Ostasiatischen Zeitschrift 1913/14 Gus fehlende Einsicht in politische Sachverhalte und bezeichnet ihn aufgrund seiner ultrakonservativen Position als „reaktionären Zensor und katonischen Sittenprediger“, als „rückständige[n] Eigenbrödler“. ${ }^{43}$ Er verurteilt Gus politische Erörterungen als antieuropäische Hetze und entzieht ihnen

34 Paquet: Vorwort (Anm. 11), S. i.

35 Hesse: Chinas Verteidigung gegen europäische Ideen (Anm. 13), S. 78.

36 Pannwitz: Die Krisis der europäischen Kultur (Anm. 16), S. 228 und S. 247.

37 Mühlestein: Europäische Reformation (Anm. 18), S. 237.

38 Vgl. Nelson: Erziehung zum Führer (Anm. 18), S. 500, sowie ders.: Ethischer Realismus (Anm. 18), S. 288.

39 Vgl. Nelson: Vorworte und Einführung zum Sammelband: Die Reformation der Gesinnung durch Erziehung zum Selbstvertrauen, in: Sittlichkeit und Bildung (Anm. 18), S. 241-245, hier S. 241.

40 Vgl. Mühlestein: Europäische Reformation (Anm. 18), S. 237-238.

41 Ebd., o. A.

42 Vgl. Hesse: Chinas Verteidigung gegen europäische Ideen (Anm. 13), S. 78-79.

43 Ludwig Rieß: Ku Hung-Ming, Chinas Verteidigung gegen europäische Ideen [Rez.], in: Ostastiatische Zeitschrift 2, 1913/1914, S. 233-235, hier S. 234. 
ihre theoretische Grundlage. ${ }^{44}$ Diese Einschätzung stellt in den 1910er Jahren zwar eine Ausnahme dar, deckt sich aber mit frühen Rezeptionszeugnissen der Jahrhundertwende ${ }^{45}$ und auch den späten der zweiten Hälfte der 1920er Jahre. ${ }^{46}$ Selbst Richard Wilhelm, einst glühender Anhänger Gus, ${ }^{47}$ distanzierte sich aus diesem Grund um 1926 entschieden von ihm. ${ }^{48}$ Insofern war eine Absicherung der ideologischen Position Gus in der Rezeption durch dessen Heroisierung nötig, sodass ein Angriff auf ihn möglichst wirkungslos blieb.

\section{Gus Selbstinszenierung49}

Gu Hongming selbst trug nicht wenig zu seinem Bild als Schüler des Konfuzius bei, ja, er machte diese Wahrnehmung allererst möglich. Nach seiner Rückkehr nach China und seinem Bekenntnis zum Konfuzianismus führte er nach außen hin ein Leben, das genau das verkörperte. Er hatte sich zu einem Lehrmeister des Konfuzianismus und zu einem entschiedenen Verfechter der Mandschu-Dynastie entwickelt. ${ }^{50}$ Selbst nach der Revolution 1911 und der Ausrufung der Republik 1912 trat er für ihre Wiedereinsetzung ein. Zudem orientierte er sich in seinem Auftreten maßgeblich an westlichen Vorstellungen eines typisch chinesischen Lebenswandels, die auf Spiritualität und Brauchtum fußten, und stellte das, was dort als authentisch galt, in China hingegen als antiquiert angesehen wurde, geradewegs zur Schau. Er praktizierte das Konkubinat und bis zu seinem Tode trug er die traditionelle Kleidung sowie den Zopf, Sinnbild der Mandschu-Herrschaft und des alten, geistigen China (Abb. 1). Dass er sich dessen Symbolkraft und Außenwirkung durchaus bewusst war, zeigt eine Unterhaltung zwischen Gu und dem

44 Vgl. ebd.

45 Vgl. ders.: Ku Hung-Ming - Papers from a Viceroy's Yamen [Rez.], in: Bulletin de l'École française de l'Extrême-Orient 3, 1903, S. 343-344.

46 Vgl. Richard Herbertz: Philosopher or Mystic?, in: The Living Age 310, 1921, S. 155-159, hier S. 156-157; Otto Franke: Adolf Reichwein, China und Europa, geistige und künstlerische Beziehungen im 18. Jahrhundert [Rez.], in: Ostastiatische Zeitschrift 1, 1924, S. 6669, hier S. 66; Ernst Viktor Zenker: Geschichte der chinesischen Philosophie, Bd. 2: Von der Han-Dynastie bis zur Gegenwart, Reichenberg 1927, S. 320-321.

47 Vgl. etwa Salome Wilhelm: Richard Wilhelm. Der geistige Mittler zwischen China und Europa. Mit einer Einleitung von Walter F. Otto, Düsseldorf/Köln 1956, S. 183-184.

48 Vgl. Wilhelm: Die Seele Chinas (Anm. 6), S. 174; ders.: Die chinesische Literatur, Potsdam 1926, S. 192.

49 Ich verweise hier auf das in der Literaturwissenschaft mittlerweile gängige Konzept der ,Autorschaftsinszenierung', das, wie hier versucht, den performativen und sozialen Aspekten der Positionierung einer Autorin, eines Autors im literarischen Feld Rechnung trägt; einen guten Überblick bieten Christoph Jürgensen / Gerhard Kaiser: Schriftstellerische Inszenierungspraktiken - Heuristische Typologie und Genese, in: Christoph Jürgensen / Gerhard Kaiser (Hg.): Schriftstellerische Inszenierungspraktiken - Typologie und Geschichte, Heidelberg 2011, S. 9-30.

50 Die Mandschu- bzw. Qing-Dynastie ist die letzte Dynastie des chinesischen Kaiserreichs. Nach über 250-jähriger Herrschaft endete sie 1911 mit der Revolution. 


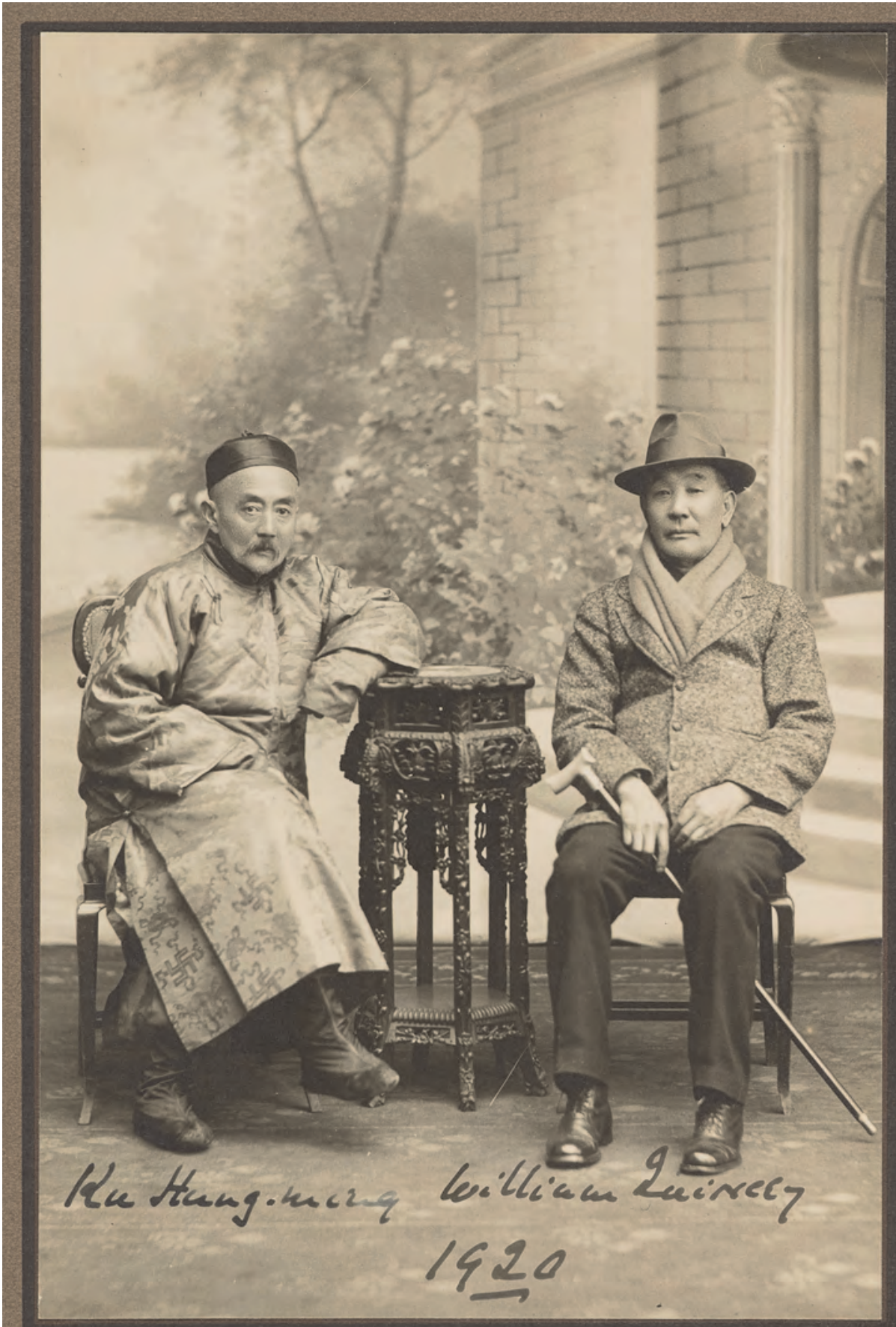

Lai Afong: Ku Hung-ming und William Quincey, 1920, Schwarzweißfotografie, 15,20 × 10,10 cm, National Galleries of Scotland, ID: PGPLA 64. 
englischen Schriftsteller William Somerset Maugham, der ihn in Peking besuchte. Über seinen Haarzopf erläutert er: „It is a symbol. I am the last representative of the old China. " ${ }^{11}$ Auch wenn Gus Lehrmeinung manche Male abgeurteilt wurde, so reichte sein an der Tradition und am Konfuzianismus ausgerichtetes Lebensmodell häufig aus, Wertschätzung und Bewunderung hervorzurufen. Hermann Graf Keyserling etwa stand Gus Kulturtheorien wie chinesischen Lehren überhaupt skeptisch gegenüber, schätzte jedoch seine Lebenshaltung. In seinem Reisetagebuch gelangt Graf Keyserling zu dem verallgemeinernden Schluss:

Je mehr ich von den Chinesen sehe, desto mehr fällt mir auf, wie uninteressant ihre Gedanken sind. Das Denken ist eben nicht ihr Eigentliches: ihr Dasein ist der Ausdruck ihrer Tiefe. So ist auch Ku Hung-Ming als Mensch viel bedeutender denn als Schriftsteller und Denker. ${ }^{52}$

Gu konnte sich - zumindest im Westen, denn in China galt er als lebender Anachronismus, er wurde als überholt kaum mehr ernst genommen oder gar angefein$\operatorname{det}^{53}$ - erfolgreich ein Image als , authentischer ${ }^{6}$ Chinese konfuzianischer Färbung aufbauen und dieses sein Leben lang aufrechterhalten. Das von Kulturskeptizismus und Eskapismus geprägte intellektuelle Klima in Westeuropa wusste Gu so für sich zu nutzen. Sowohl durch die Verbreitung der klassischen chinesischen Kultur in seinen Schriften wie auch durch seine als traditionell chinesisch wahrgenommene Lebensweise konnte er dieses Bild glaubhaft vermitteln. Seine Leser betonten denn auch die ,chinesische' Art seiner Betrachtungen, besonders dann, wenn es darum ging, Denkfehler zu entschuldigen, wie in diesen beiden Rezensionen:

Kus Buch ist voller Wiederholungen und Widersprüche; nicht weil es seinem Denken an Konzentration fehlte, sondern weil er minutiös denkt, wie der chinesische Maler die Haare eines Tigers, die Federn einer Wachtel und die Halme und Knötchen der Halme in einem Bestand von Schilf sieht[.] ${ }^{54}$

Ku Hungming ist zum Schriftsteller für die Westländer geworden. Doch ist er dabei Chinese geblieben und hat sich die charakteristischen Eigenschaften des schriftstellernden Chinesen bewahrt. Die große Zahl seiner Zitate aus europäischen Werken, die Neigung zu schematisieren statt zu analysieren, das Gefallen an Wortbildern, in welche die häufig in die Breite statt in die Tiefe gehenden Gedankengänge hineingezwängt werden, eine gewisse Eitelkeit nach außen statt nach innen, das alles sind Merkmale, die ihn als Kind seines Landes verraten [...]. ${ }^{55}$

51 Zit. nach: Tianhu Hao: Ku Hung-Ming, an Early Chinese Reader of Milton, in: Milton Quarterly 39.2, 2005, S. 93-100, hier S. 98.

52 Graf Keyserling: Das Reisetagebuch eines Philosophen (Anm. 14), S. 394.

53 Dies geht aus der Rezension von Dewall: Ku Hung-Ming (Anm. 13), S. 371, hervor; vgl. außerdem Müller: Gu Hongming (Anm. 3), S. 8; Du: Gu Hongming as a Cultural Amphibian (Anm. 3), S. 716; ders.: Gu Hongming's Eccentric Chinese Odyssey (Anm. 3), S. 12-14.

55 Dewall: Ku Hung-Ming (Anm. 13), S. 371. 
Gu Hongmings Selbstinszenierung als traditioneller Chinese, sein Kulturkonservatismus wie auch sein daran geschultes Auftreten prädestinierten ihn zum Sprachrohr für eine ethnographische Kritik an der europäischen Moderne und zum Vertreter eines fremden, nachahmenswerten Lebens- und Gesellschaftsmodells. Zugleich aber - und dies ist nicht zu unterschätzen - bot Gu durch seine europäische Bildung, die sich sowohl in der sprachlichen Gestaltung als auch im Inhalt seiner Texte niederschlug, genügend Anschlussmöglichkeiten für das Publikum im Westen. Seine Werke verfasste Gu auf Englisch und er baute zahlreiche Referenzen auf klassische wie moderne Texte der europäischen Tradition ein. Er zitierte die Bibel, Shakespeare und Goethe sowie die britischen Denker und Dichter des viktorianischen Zeitalters Matthew Arnold, Thomas Carlyle, John Ruskin und Alfred Tennyson. ${ }^{56}$ Unterschiede zwischen Ost und West ebnete Gu kurzerhand ein: Der hebräischen Bibel entsprach für ihn in China Four Books and Five Classics, in den Lehren von Konfuzius und dessen Nachfolger Mengzius fand er ähnliche Ideen wie bei Carlyle, und die Kaiserinwitwe Cixi stellte er Queen Victoria gegenüber. ${ }^{57}$ Insgesamt parallelisierte er die gesellschaftlichen und literarhistorischen Entwicklungen beider Kulturkreise, etwa in seinem Aufsatz The Story of a Chinese Oxford Movement, in dem er die Gruppe um den Gouverneur Zhang Zhidong, für den Gu tätig gewesen war, und die Oxford-Bewegung des Kardinal Newman des 19. Jahrhunderts nebeneinanderstellt. Einerseits erleichterte dieses Vorgehen westlichen Lesern den Zugang zu seinen Texten und damit den politischen, gesellschaftlichen und kulturellen Eigenheiten Chinas, andererseits fanden sie in dem fremden Text, an dem sie stets das Andere hervorhoben, das Eigene. So ist es doch bezeichnend, wenn Pannwitz ihn zum „chinesische[n] demosthenes ${ }^{\text {"58 }}$ stilisiert und ihn so in die westeuropäische Tradition einbürgert,

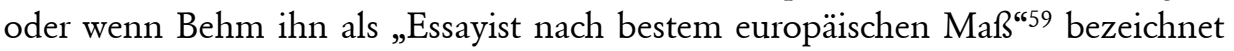
und ihn mit dem konservativen deutschen Kulturphilosophen Paul de Lagarde gleichsetzt, der für eine Rückbesinnung auf spezifisch christlich-germanische Werte eintrat. ${ }^{60}$

56 Dies wird von seinen Rezensenten stets betont, z. B. Paquet: Vorwort (Anm. 11), S. i-ii; Behm: Ein chinesischer Spiegel (Anm. 13), S. 864; Nelson: Geleitwort des Übersetzers (Anm. 12), S. 3; vgl. Du: Gu Hongming as a Cultural Amphibian (Anm. 3), S. 724; ders.: Gu Hongming's Eccentric Chinese Odyssey (Anm. 3), S. 26-28; Müller: Gu Hongming (Anm. 3), S. 9. Zu Gus Kenntnis von Goethes Werk vgl. Wei Maoping: Goethe in China Mit besonderer Berücksichtigung der Umsetzung von Ku Hung-Ming sowie einem Blick in die Zukunft, in: Jahrbuch für internationale Germanistik 47.1, 2015, S. 89-99. Vgl. Du: Gu Hongming as a Cultural Amphibian (Anm. 3), S. 730 und S. 734; ders.: Gu Hongming's Eccentric Chinese Odyssey (Anm. 3), S. 35-36.

59 Behm: Ein chinesischer Spiegel (Anm. 13), S. 864.

60 Vgl. ebd., S. 867-868. 


\section{Fazit}

Gu Hongming trägt aus Sicht seiner Rezipienten den Stempel des fremden Landes, was ihm Einsicht in die konfuzianische Philosophie und einen schärferen Blick auf Europa verleihe. Zugleich aber kreieren seine europäischen Leser eine Wahlverwandtschaft mit ihm, um zu beweisen, dass Gus Schreiben dem europäische Standard entspricht. Das Zusammenspiel aus Assimilierung und Alienisierung Gus, aus Vertrautheit und Fremdheit, Nähe und Distanz, verbürgt auf der seinen Seite den als notwendig erfahrenen ethnographischen Blick auf das Eigene und ermöglicht auf der anderen seine Aufnahme in den europäischen Kanon, in welchem er für seinen Blick von außen eine exzeptionelle Stellung erhält. Die Heroisierung zum Schüler des Konfuzius, die Gu durch seine Selbstinszenierung als traditionsverbundener Chinese und konfuzianischer Prophet stützt, sichert diese Position gegen mögliche Kritik ab und garantiert die Geltung und Wirksamkeit seiner Anschauung. Im Zentrum der deutschen und österreichischen Rezeption insbesondere durch kulturkonservative Intellektuelle stand $\mathrm{Gu}$ mithin nicht nur als Kulturvermittler, sondern als Kulturheros, der - ähnlich dem ,grand homme ${ }^{61}$ - für seine geistige Leistungen als Kritiker, Ideengeber, Eingeweihter und Vorbild im Kontext der europäischen Kulturkrise gewürdigt wurde.

\section{Abbildungsnachreis}

Abb. 1: National Galleries of Scotland.

61 Zum ,grand homme‘ vgl. Benjamin Marquart: Grand homme, in: Ronald G. Asch u. a. (Hg.): Compendium heroicum, publiziert vom Sonderforschungsbereich 948 „Helden Heroisierungen - Heroismen" der Universität Freiburg, Freiburg 1. Januar 2018, DOI: 10.6094/heroicum/grand-homme. 
